\title{
Simultaneous identification and determination of flavonoids in Dendrobium officinale
}

\author{
Chunhua Zhou ${ }^{1 \dagger}$, Zhenshan Xie ${ }^{1 \dagger}, Z^{2}$ houxi Lei ${ }^{1}$, Yuechun Huang ${ }^{2^{*}}$ and Gang Wei ${ }^{1^{*}}$ (D)
}

\begin{abstract}
Background: The quality of material medicine resources has had a considerable impact on the development of the health industry, which has created a bottleneck for traditional Chinese medicine (TCM). Dendrobium officinale, which has been widely used for health prevention in TCM, has become a high-nutritive health food that is strongly recommended by many white-collar workers and people who pay more attention to their health. The aim of this study was to develop a method to authenticate and evaluate $D$. officinale from different origins via simultaneous qualitative and quantitative analyses of flavonoid glycosides. Ultra-high-performance liquid chromatography-electrospray ionization/ mass spectrometry was used for the structural elucidation of the compounds.
\end{abstract}

Results: 9 characteristic peaks, including those representing 7 flavonoid C-glycosides and 2 flavonoid O-glycosides, were identified. Additionally, the contents of 5 representative flavonoid glucosides in 25 batches of $D$. officinale from different sources were determined. To further investigate the different sources of the 25 batch samples, principal component analysis (PCA) and hierarchical cluster analysis (HCA) were carried out. A study on the methodology revealed that all results were reliable.

Conclusions: This method is an efficient tool for the rapid identification of the different geographical origins of $D$. officinale and provides references for the quality evaluation of other natural products.

Keywords: Dendrobium officinale, Different origins, Flavonoid glucosides, Identification, Quantitative analysis

\section{Introduction}

The Dendrobium genus is one of the largest genera of Orchidaceae [1]. There are thousands of species of Dendrobium all over the world [2]. Dozens of species, including Dendrobium officinale, Dendrobium nobile, Dendrobium huoshanense and Dendrobium chrysanthum, are grown in China [3]. Among all of these Dendrobium species, Dendrobium officinale is one of the most popular for its functions in TCM, such as tonifying the stomach, promoting fluid, nourishing yin and clearing

\footnotetext{
*Correspondence: huangyuechun@163.com; weigang021@outlook.com ${ }^{\dagger}$ Chunhua Zhou and Zhenshan Xie contributed equally to this work ${ }^{1}$ School of Pharmaceutical Sciences, Guangzhou University of Chinese Medicine, 232 Outer Circle Road East, Guangzhou Higher Education Mega Center, Guangzhou 510006, China

${ }^{2}$ The First Affiliated Hospital, Guangzhou University of Chinese Medicine, Guangzhou 510405, Guangdong, People's Republic of China
}

heat $[4,5]$. The fresh stem of $D$. officinale can be orally consumed directly, and it can also be used as a soup stock or tea. Meanwhile, modern pharmacology studies have indicated that $D$. officinale has some beneficial bioactivities, such as anti-oxidant, anti-tumor, hypoglycemic, and hypoglycemic activities and gastrointestinal regulatory functions [6-8].

Generally, the quality of genuine regional remedies are outstanding [9]. D. officinale from the Danxia landform region has become a genuine medicinal material since the Northern and Southern Dynasties of China, which were approximately 1500 years ago. Since then, the herbal medicine $D$. officinale has mainly been distributed in some Danxia landform regions located in the Fujian and Guangdong provinces, and the Danxia landform area was the first main habitat of $D$. officinale. However, since the Song Dynasty, the Guangnan area (consisting of the 
Yunnan and Guangxi provinces) and Zhejiang province became the two main habitats of this herb. After considerable consultation of ancient herbal documents and on-the-spot investigation, we discovered that $D$. officinale that grew in the abovementioned 3 habitats were different from each other in character and shape (as shown in Fig. 1). Thus, we assumed that the content and types of chemical compounds in this herb may be different. The main active ingredients of $D$. officinale are phenols and polysaccharides [10-13]. To date, studies on D. officinale have mainly focused on the polysaccharides [14-16]. However, polysaccharides are ubiquitous in Dendrobium species. Flavonoids are a widespread group of phytochemicals with diverse biological functions and significant substances in plants that not only play a key role in the pharmaceutical industry but also serve as excellent chemical markers for quality control of medicinal plants [17-19]. Several reports have studied the flavonoids in different parts of $D$. officinale [20]. However, no reports have been published on the effects of the producing region on the flavonoids in $D$. officinale, and this is not comprehensive to study quality control. By comparing the constituents of $D$. officinale from the three main genuine producing regions and by searching for common specific components, the chemical differences in the different producing regions were revealed. This was critical for the synthetic evaluation of $D$. officinale.

To establish a comprehensive evaluation system for D. officinale, we utilized UHPLC-ESI-MS/MS fingerprint chromatography. Then, the chemical markers were identified, and the contents of 25 batch samples were collected from the Danxia landform region in Zhejiang province and tested. Furthermore, principal component analysis (PCA) and hierarchical cluster analysis (HCA) were utilized to analyze the different sources of $D$. officinale. These results showed that this method could be successfully used for identifying specific discriminating markers to identify $D$. officinale from different geographical environments and to improve the quality evaluation system of $D$. officinale.

\section{Experimental}

\section{Chemicals and reagents}

Apigenin-6,8-di-C- $\beta$-D-glucoside, isoviolanthin and apigenin-6-C- $\beta$-D-xyloside- 8 -C- $\beta$-D-Glucoside were isolated from the leaves of $D$. officinale via preparative liquid chromatography as reference substances for the experiments. Rutin, naringin and schaftoside were obtained from the National Institute Control of Pharmaceutical and Biological Products (Guangzhou, China). The purity of all compounds mentioned above was over $96 \%$, and the compounds were of HPLC grade; their chemical structures were identified by comparing their UV, IR, ESI/MS, and NMR spectra with other published reports.

HPLC grade methanol was purchased from Merck (Darmstadt, Germany). Ultrapure water was prepared using a Milli-Q water purification system (MA, USA). Analytical grade methanol and ammonium acetate $\left(\mathrm{CH}_{3} \mathrm{CO}_{2} \mathrm{NH}_{4}\right)$ were obtained from Damao Chemical Corporation, Tianjin, China. Tetrahydrofuran was purchased from Mreda (USA).

Twenty-five samples of fresh, mature D. officinale stems were collected from different regions of China in the Danxia landform area (Fujian, Guangdong, and Jiangxi),

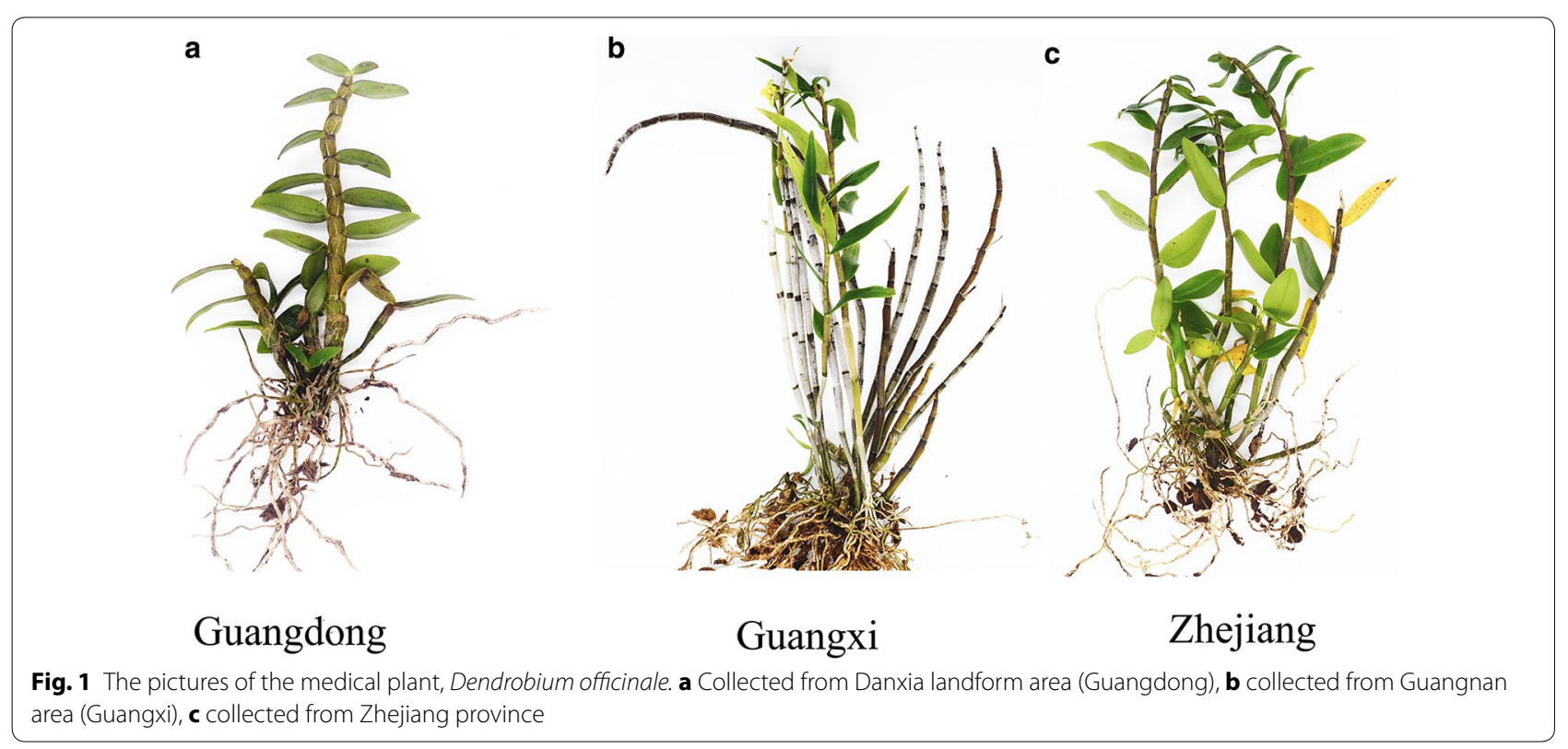


Guangnan area (Guangxi and Yunnan) and Zhejiang province. Of these, 3 batches were from Guangdong province (No. GD1-GD3), 3 batches were from Jiangxi province (No. JX1-JX3), 1 batch was from Fujian province (No. FJ), 8 batches were from Zhejiang province (No. ZJ1-ZJ8), 5 batches were from Yunnan province (No. YN-YN5), and 5 batches were from Guangxi province (No. GX1-GX5).

\section{Preparation of standard solutions and sample preparation} A set of standard solutions were prepared by appropriate dilution of the stock solution with methanol. They were then diluted to construct different calibration plots in the following ranges: $9.25-1850 \mathrm{ng} / \mathrm{mL}$ for apigenin6,8-di-C- $\beta$-D-glucoside, $8.43-1686 \mathrm{ng} / \mathrm{mL}$ for apigenin6-C- $\beta$-D-xyloside- $8-C$ - $\beta$-D-glucoside, $7.14-1428 \mathrm{ng} / \mathrm{mL}$ for schaftoside, $16.5-3300 \mathrm{ng} / \mathrm{mL}$ for rutin, and 120 $2390 \mathrm{ng} / \mathrm{mL}$ for isoviolanthin.

The samples were dried and ground into powder; $1.0 \mathrm{~g}$ powder was placed in $100 \mathrm{~mL}$ Erlenmeyer flasks and ultrasonically extracted twice for 45 min each with $50 \mathrm{~mL}$ methanol. The total extract was concentrated and evaporated to dryness, and the residue was re-dissolved with an adequate amount of methanol: water $(80: 20, \mathrm{v} / \mathrm{v})$. Then, the solution was transferred to a $2 \mathrm{~mL}$ volumetric flask and diluted to a constant volume $(10 \mathrm{~mL})$. Prior to injection, all solutions were filtered through a $0.22 \mu \mathrm{m}$ microporous membrane. All the solutions were stored in a refrigerator at $4{ }^{\circ} \mathrm{C}$ before analysis.

\section{Qualitative and quantitative analysis}

Quantitative analyses were performed using a UHPLC system equipped with a vacuum degasser, quaternary pump, auto-sampler and ultraviolet detector (Thermo Separation Products Inc., Riviera Beach FL, USA). All data were processed on a Finnigan Xcalibur 2.0 advanced chromatography workstation (Thermo Quest Corporation, San Jose, CA, USA). The studies were conducted on a Hypersil GOLD C18 $(100 \times 2.1 \mathrm{~mm}$ ID, $1.9 \mu \mathrm{m}$, Thermo, USA) with a suitable guard column (C18, ODS, $1.9 \mu \mathrm{m}, 4.0 \times 3.0 \mathrm{~mm}$ ). The mobile phase consisted of methanol (A) and a $10 \mathrm{mM}(\mathrm{v} / \mathrm{v})$ ammonium acetate aqueous solution (B) with a linear gradient elution at a flow rate of $200 \mu \mathrm{L} / \mathrm{min}$. The elution program was carried out according to the following profile: $0-10 \mathrm{~min}$, 20-23\% A; $10-15$ min, $23-26 \%$ A; $15-16$ min, 26-30\% A; $16-25 \mathrm{~min}, 30-35 \%$ A; $25-30 \mathrm{~min}, 35-42 \%$ A; 30-35 min, $42-35 \%$ A. The column temperature was maintained at $30^{\circ} \mathrm{C}$, and the sample injection volume was $3 \mu \mathrm{L}$. The detection wavelength was $340 \mathrm{~nm}$. MS analysis was performed on a Thermo Finnigan LCQ FLEET equipped with an ion trap mass spectrometer with an electrospray ionization interface and an ultraviolet detector. Nitrogen was used as the sheath and auxiliary gas, and helium was used as the collision gas. The ESI/ MS spectra were acquired in both positive and negative ion modes. The ESI source conditions were as follows: spray voltage of $2800 \mathrm{~V}$ in negative ion ESI mode, $3500 \mathrm{~V}$ in positive ion ESI mode, capillary temperature of $350{ }^{\circ} \mathrm{C}$, sheath gas flow rate of 30 (arbitrary units), auxiliary gas flow rate of 10 (arbitrary units), and scan range for both MS and MS/MS between $m / z 150$ and 1000 .

Quantitative analyses were run on an Agilent 1100 system. Chromatographic separations were carried out on a Kromasil 100-5 C18 $(250 \times 4.6 \mathrm{~mm}, 5.0 \mu \mathrm{m})$ maintained at $30^{\circ} \mathrm{C}$. The mobile phases consisted of water containing tetrahydrofuran: acetonitrile: methanol (10:22:5) (A) and $0.05 \%$ phosphoric acid (B), and the elution gradient was set as follows: 0-10 min, $10-11 \%$ A; $10-25$ min, $11-11.5 \%$ A; $25-32 \mathrm{~min}$, $11.5-12 \%$ A; 32-42 min, $12-12.5 \%$ A; $42-52$ min, $12.5-$ $13.5 \%$ A; $52-75 \mathrm{~min}, 13.5-14 \% \mathrm{~A}$. The flow rate was $1 \mathrm{~mL} /$ min, and the detection wavelength was $340 \mathrm{~nm}$.

\section{Method validation}

The 5 standard solutions were diluted to six different concentrations with methanol to investigate the linearity. The concentration of the standard solutions was represented on the $\mathrm{X}$-axis $(\mathrm{X})$, the chromatographic peak area was on the $\mathrm{Y}$-axis $(\mathrm{Y})$, and the results were analyzed by linear regression statistics. The repeatability was evaluated by carrying out six replicate analyses of the same sample (YN4). The $\mathrm{RSD}_{\mathrm{S}}$ for the retention time and peak area were calculated as measures of repeatability.

The precision was investigated by analyzing the sample on the same day (intra-day) and between 3 consecutive days (inter-day). For precision absorption of the sample solution of $D$. officinale from Yunnan (YN4), the samples were analyzed 6 times continuously, and the $\mathrm{RSD}_{\mathrm{S}}$ for the retention time and peak area were calculated to evaluate the intra-day and inter-day precision. To evaluate the stability of the sample, selected sample (YN4) was analyzed at room temperature at $0,2,4,6,8,12$, and $24 \mathrm{~h}$ after preparation, and the stability was expressed by the $\mathrm{RSD}_{\mathrm{S}}$ for the retention time and peak area.

The recoveries of the 5 compounds were determined by spiking the sample (YN4) with suitable amounts (approximately $100 \%$ of the contents) of the standard compounds that were previously determined. The actual amounts in relation to the theoretically present amounts were expressed as a percentage of the recovery.

\section{Chemometric analysis}

To distinguish the relatively homogeneous groups of the 25 D. officinale from different origins, the HCA multivariate analysis technique was performed using SPSS software (SPSS 23.0 for Windows, SPSS Inc., USA). 


\section{Results and discussion}

\section{Characterization of flavonoid glycosides}

UHPLC-ESI-MS/MS was adopted to characterize the target constituents of $D$. officinale. Both positive and negative ion modes were utilized to ionize the flavonoid glycosides, and negative mode ESI was found to be sensitive for flavone glycosides of $D$. officinale, which showed the $[\mathrm{M}-\mathrm{H}]^{-}$deprotonated ions in the negative mode ESI-MS spectra for all the flavonoid glycoside ingredients. The product ion scans of the 9 flavonoid glycosides re shown in Fig. 2. By comparing the retention times from the UV and ESI-MS ${ }^{\mathrm{n}}$ spectra with literature data, we identified and deduced the possible structures of the 9 main constituents, including flavonoid $\mathrm{O}$-glycosides and flavonoid $\mathrm{C}$-glycosides. The TIC chromatogram is shown in Fig. $2 \mathrm{~b}$. The retention times $\left(t_{R}\right)$, MS and $M^{2}$ spectral data and identification of the flavonoids are listed in Table 1. Some compounds were unambiguously identified by comparing them with the reference compound. However, because of the unavailability of authentic compounds, some of the peaks could only be tentatively assigned.

\section{Characterization of flavonoid C-glycosides}

In this study, 7 flavonoid C-glycosides were identified in D. officinale by peaks $1,2,3,4,5,7$ and 8 , and the characteristic fragment patterns of flavonoid C-glycosides resulted from the cleavage of the glucosyl as follows: a series of fragment ions arising from the loss of $[(\mathrm{M}-\mathrm{H})$ $\left.60]^{-}, \quad[(\mathrm{M}-\mathrm{H})-90]^{-},[(\mathrm{M}-\mathrm{H})-120]^{-}, \quad[(\mathrm{M}-\mathrm{H})-90-120]^{-}\right]$, $[\mathrm{M}-\mathrm{H}-2 \times 120]^{-},[(\mathrm{M}-\mathrm{H})-120-\mathrm{CO}]^{-}$, and $[(\mathrm{M}-\mathrm{H})-2 \times 120-$ $2 \mathrm{CO}]^{-}$were the major fragmentation pathways in the MS or MS/MS. The flavonoid C-glycosides in D. officinale

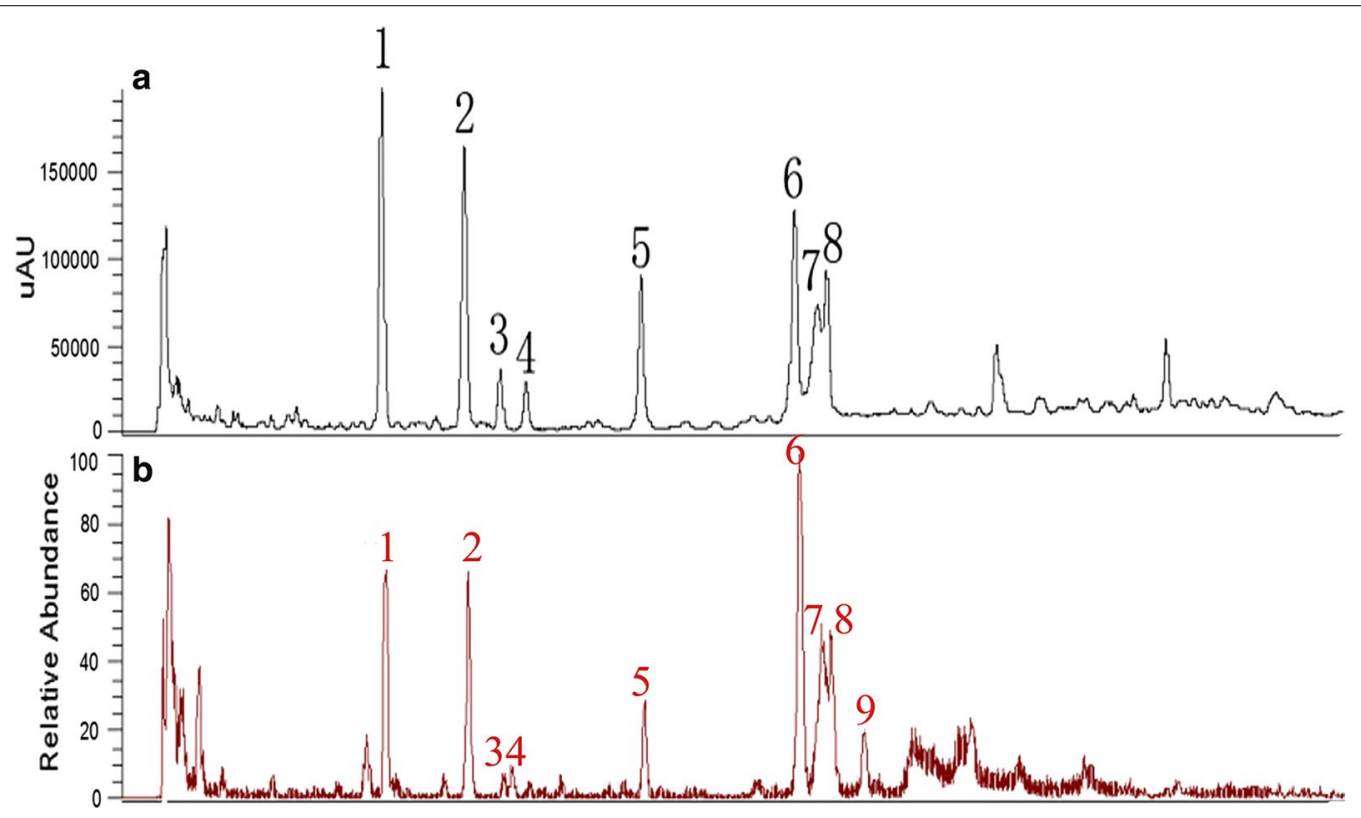

Fig. 2 UHPLC-UV chromatograms of Dendrobium officinale (a), The UHPLC-ESI/MS (TIC) fingerprint of Dendrobium officinale (b)

Table 1 MS date for characterization of compounds in Dendrobium officinale by UHPLC-ESI-MS/MS

\begin{tabular}{|c|c|c|c|c|}
\hline Peak no & $\mathrm{RT}(\min )$ & Negative ions (m/z) & $M^{n}$ & Identification \\
\hline 1 & 7.45 & $593[\mathrm{M}-\mathrm{H}]^{-}$ & $M^{2}: 503.19,473.01,383.11,353.09$ & Apigenin-6,8-di-C- $\beta$-D-glucoside \\
\hline 2 & 9.82 & $563[\mathrm{M}-\mathrm{H}]^{-}$ & $M^{2}: 503.08,473.04,443.08,383.10,353.07$ & Apigenin-6-C- $\beta$-D-xyloside-8-C- $\beta$-D-glucoside \\
\hline 3 & 10.85 & $563[\mathrm{M}-\mathrm{H}]^{-}$ & MS $^{2}: 503.07,473.03,443.10,383.06,353.05$ & Isoschaftoside \\
\hline 4 & 11.58 & $563[\mathrm{M}-\mathrm{H}]^{-}$ & 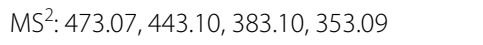 & Schaftoside \\
\hline 5 & 14.87 & $563[\mathrm{M}-\mathrm{H}]^{-}$ & $M^{2}: 473.09,443.10,383.18,353.12$ & Apigenin-6-C- $\beta$-D-glucoside-8-C- $\beta$-D-xyloside \\
\hline 6 & 19.28 & $609[\mathrm{M}-\mathrm{H}]^{-}$ & $\begin{array}{l}\text { MS }^{2}: 301.04,271.09 \\
M^{3}: 271.07,255.08,179.00,151.01\end{array}$ & Rutin \\
\hline 7 & 20.04 & $577[\mathrm{M}-\mathrm{H}]^{-}$ & MS $^{2}: 457,383$ & Isoviolanthin \\
\hline 8 & 20.18 & $577[\mathrm{M}-\mathrm{H}]^{-}$ & $\mathrm{MS}^{2}: 457,383$ & Violanthin \\
\hline 9 & 21.27 & $579[\mathrm{M}-\mathrm{H}]^{-}$ & $M^{2}: 417$ & Naringin \\
\hline
\end{tabular}


usually are present on the $\mathrm{C}-6$ and $\mathrm{C}-8$ position. Furthermore, glycosides on the $\mathrm{C}-6$ position exhibited more fragmentation than those from the $\mathrm{C}-8$ position. In the positive ESI/MS of $D$. officinale, the base peaks always appeared as $[\mathrm{M}+\mathrm{H}]^{+}$and $[\mathrm{M}+\mathrm{Na}]^{+}$ions, which were further fragmented by the successive losses of one molecule of $\mathrm{H}_{2} \mathrm{O}$, leading to the product ions $[\mathrm{M}+\mathrm{H}-18]^{+}$. The trials showed that the negative ion mode was more sensitive than the positive ion mode.

Peak 1 represented $[\mathrm{M}-\mathrm{H}]^{-}$at $m / z$ 593. The fragment ion peaks shown in Fig. 3a, including $473[(\mathrm{M}-\mathrm{H})-120]^{-}$, 353 [(M-H)-120-90] $]^{-}$, were assigned to apigenin-6,8-diC- $\beta$-D-glucoside by comparing with the standard. Peaks 2 and 3 represented a molecular ion $[\mathrm{M}-\mathrm{H}]^{-}$at $m / z 563$, which produced a similar $\mathrm{MS}^{2}$ base peak at $m / z 473$ [(M$\mathrm{H})-90]^{-}$and an $\mathrm{MS}^{3}$ base peak at $m / z 353$ [(M-H-90$120]^{-}$. Upon comparison with the standards, peak 2 was established to be due to apigenin-6-C- $\beta$-D-xyloside-8-C$\beta$-D-glucoside, whereas peak 3 was identified as isoschaftoside, as shown in Fig. 3b. Peaks 4 and 5 (in Fig. 3c) also represented the same molecular ion $[\mathrm{M}-\mathrm{H}]^{-}$at $m / z 563$, yielding product ions at $m / z 443[(\mathrm{M}-\mathrm{H})-120]^{-}$and 353 [(M-H)-120-90] ${ }^{-}$. Meanwhile, fragment ions at $\mathrm{m} / z 503$

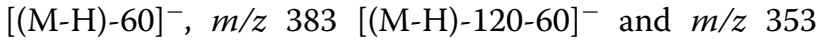
$[(\mathrm{M}-\mathrm{H})-120-90]^{-}$were also present. Moreover, these findings were consistent with literature data [21]. Compound 4 was unambiguously identified as schaftoside by comparing with the standard, and peak 5 was assigned to apigenin-6-C- $\beta$-D-glucoside- $8-C-\beta$-D-xyloside. Peaks 7 and 8 were identified as representing isoviolanthin and violanthin (Fig. 3d), which had similar molecular ions
[M-H] $]^{-}$at $m / z 577$ and the same fragment ions at 457 $[(\mathrm{M}-\mathrm{H})-120]^{-}$.

\section{Characterization of flavonoid O-glycosides}

The fragmentation behaviors of peak 6 with the loss of $\mathrm{m} / z 308,146,162$, and 176 revealed the possible presence of flavonoid $O$-glycosides, whereas $308 \mathrm{Da}$ was confirmed to be a typical fragment of rutinose. Furthermore, peak 6 molecular ions at $609.34[\mathrm{M}-\mathrm{H}]^{-}$and $610.82[\mathrm{M}+\mathrm{H}]^{+}$, which produced fragments at $m / z 301[(\mathrm{M}-\mathrm{H})-308]^{-}, m / z$ $300[(\mathrm{M}-2 \mathrm{H})-308]^{-}$, and $m / z 303$ in positive ion mode, were evidence of quercetin. Peak 6 was assigned to rutin, as shown in Fig. 3e, which was unambiguously identified via comparison with the reference standard. Peak 9 showed an $[\mathrm{M}-\mathrm{H}]^{-}$ion at $m / z 579$ and a fragment ion at $m / z 417$ due to the successive loss of $162 \mathrm{Da}$ and was identified as naringin (shown in Fig. 3f).

\section{Method validation}

As listed in Tables 2 and 3, all the calibration curves showed good linearity in their corresponding ranges for the 5 analyses $\left(R^{2}>0.999\right)$. The intra-day RSDs and interday RSDs of the 5 compounds were $0.50-2.57 \%$, and $0.80-2.45 \%$, respectively. The values for repeatability and stability were less than $2.60 \%$, indicating that the sample possessed excellent stability over $24 \mathrm{~h}$. The average recovery of the assay was between 100.72 and $102.11 \%$, with RSDs of $0.93-2.93 \%$. All the data indicated that the developed method is satisfactory for the qualitative and quantitative analysis of $D$. officinale.
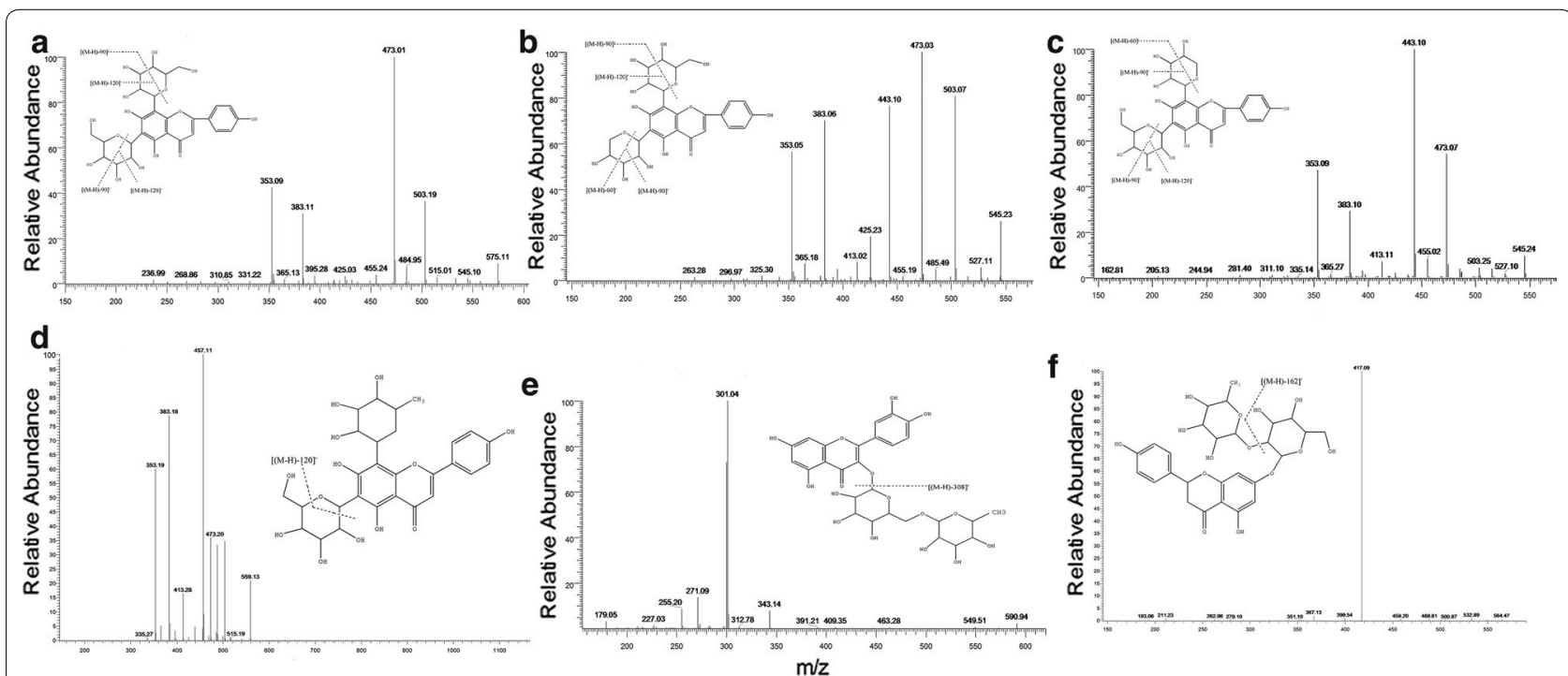

Fig. 3 Chimical structures and MS/MS spectra of 6 flavonoids, Apigenin-6,8-di-C-ß-glucoside (a), Isoschaftoside (b), Schaftoside (c), Violanthin (d), Rutin (e), Naringin (f) 
Table 2 Regression equation, correlation coefficient $\left(\mathbf{R}^{2}\right)$, linear range for 5 representative compounds from Dendrobium officinale

\begin{tabular}{llll}
\hline Compounds & Regression equation & $\mathbf{R}^{\mathbf{2}}$ & Linear range $(\mathbf{n g} / \mathbf{m L})$ \\
\hline Apigenin-6,8-di-C- $\beta$-D-glucoside & $y=1770.70 x+11.105$ & 0.9997 & $9.25-1850$ \\
Apigenin-6-C- $\beta$-D-xyloside-8-C- $\beta$-D-glucoside & $y=1889.51 x+5.476$ & 0.9998 & $8.43-1686$ \\
Schaftoside & $y=2046.54 x+14.891$ & 0.9998 & $7.14-1428$ \\
Isoviolanthin & $y=1719.51 x-4.4021$ & 0.9999 & $119.51-2390$ \\
Rutin & $y=1202.72 x+11.916$ & 0.9998 & $16.50-3300$ \\
\hline
\end{tabular}

Table 3 Repeatability, intraday and interday precisions, and stability, recovery of 5 representative compounds from Dendrobium officinale

\begin{tabular}{|c|c|c|c|c|c|c|c|c|c|}
\hline \multirow[t]{2}{*}{ Compounds } & \multirow{2}{*}{$\begin{array}{l}\text { Repeatabil- } \\
\text { ity RSD (\%) } \\
(n=6)\end{array}$} & \multicolumn{2}{|l|}{ Precisions } & \multirow{2}{*}{$\begin{array}{l}\text { Stability } \\
\text { RSD (\%) } \\
(n=6)\end{array}$} & \multicolumn{5}{|l|}{ Recovery (\%) } \\
\hline & & $\begin{array}{l}\text { Intra-day } \\
(n=6)\end{array}$ & $\begin{array}{l}\text { Inter-day } \\
(n=3)\end{array}$ & & Content $(\mu \mathrm{g})$ & Spiked $(\mu \mathrm{g})$ & Found $(\mu g)$ & $\operatorname{mean} \pm S D$ & RSD (\%) \\
\hline $\begin{array}{l}\text { Apigenin-6,8- } \\
\text { di-C- } \beta-D- \\
\text { glucoside }\end{array}$ & 0.61 & 0.50 & 0.80 & 0.43 & 21.62 & 22.21 & 44.62 & 101.54 & 1.24 \\
\hline $\begin{array}{l}\text { Apigenin-6-C- } \\
\beta-D-x y l o s i d e- \\
\text { 8-C- } \beta-D- \\
\text { glucoside }\end{array}$ & 1.10 & 1.31 & 1.28 & 1.31 & 14.51 & 15.20 & 29.93 & 101.04 & 0.93 \\
\hline Schaftoside & 2.60 & 1.84 & 2.32 & 2.13 & 2.90 & 2.81 & 5.90 & 102.11 & 2.93 \\
\hline Isoviolanthin & 1.38 & 1.37 & 1.65 & 1.45 & 39.37 & 39.44 & 78.95 & 100.81 & 1.16 \\
\hline Rutin & 1.50 & 2.57 & 2.45 & 2.190 & 6.61 & 6.32 & 12.91 & 100.72 & 1.84 \\
\hline
\end{tabular}

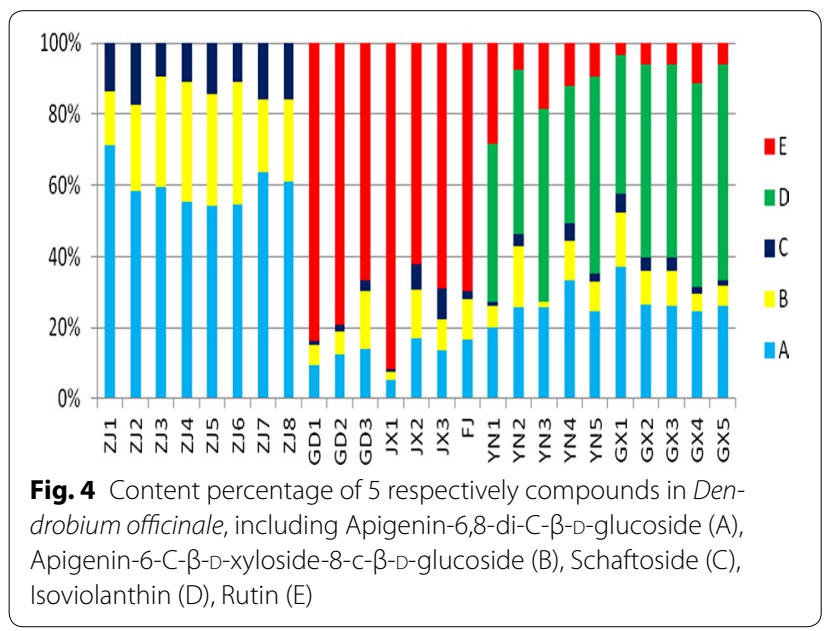

Sample quantitative analysis

There are several components in D. officinale that were identified, such as apigenin-6,8-di-C- $\beta$-D-glucoside, isoschaftoside, schaftoside, violanthin, isoviolanthin, rutin, apigenin-6-C- $\beta$-D-xyloside- $8-C-\beta$-D-glucoside, and apigenin- $6-C-\beta-D$-glucoside- $8-C-\beta-D-x y l o s i d e$ Among these components, the contents of the 5 representative compounds were determined. The content analysis was performed to observe batch-to-batch variations for samples collected from different regions, as shown in Fig. 4. Although some differences exist in the composition of samples from different producing regions, the 25 batches samples could be divided into 3 categories. The results are consistent with our previous research that the production locations of $D$. officinale could be divided into three regions [22]. Apigenin-6-C- $\beta$-D-xyloside- $8-C-\beta-D-$ glucoside, apigenin-6,8-di-C- $\beta$-D-glucoside and schaftoside are the common components that were found in all batches, whereas isoviolanthin and rutin are two marker ingredients that can be used to distinguish the source of this tonic medicine. D. officinale from Zhejiang province does not contain violanthin or rutin; however, the content of apigenin-6,8-di-C- $\beta$-D-glucoside is particularly high. Additionally, $D$. officinale from the Danxia landform region (Guangdong, Jiangxi, Fujian) has a high concentration of rutin but does not contain violanthin. In particularly, D. officinale growing in Guangxi and Yunnan contains violanthin. Because Yunan and Guangxi are geographically close to each other, the climate and environment of these two locations are similar, which may explain the reason why the compositions of the herb from these two provinces were almost identical. There are typical Danxia landform regions in Guangdong, 
Fujian and Jiangxi provinces, and the ultraviolet radiation is more intense. Consequently, the concentration of rutin is higher. The Zhejiang native species is of special provenance, and the chemical composition is different from those with other origins.

The validated method was successfully applied to determine the 5 representative compounds in the 25 batches of D. officinale, and the results are shown in Table 4 . The average total contents of apigenin-6,8-di-C- $\beta$-Dglucoside $(71.04 \mu \mathrm{g} / \mathrm{g})$ in the samples from Zhejiang were the highest, followed by those for the Danxia landform area (Fujian, Guangdong and Jiangxi) $(61.42 \mu \mathrm{g} / \mathrm{g})$ and the Guangnan area (Guangxi and Yunan) $(59.84 \mu \mathrm{g} / \mathrm{g})$. Among the 5 detected compounds, the concentration of rutin in the herbs from the Danxia landform area was the highest (Fujian, Guangdong and Jiangxi), with an average content of $375.87 \mu \mathrm{g} / \mathrm{g}$. The average content of rutin in the samples from the Guangnan area was $29.30 \mu \mathrm{g} / \mathrm{g}$. Rutin was not present in the Zhejiang native species. Isoviolanthin could be detected only in the samples from the Guangnan, area with an average content of $112.89 \mu \mathrm{g} / \mathrm{g}$.
In contrast, schaftoside was detected in the herbs from all regions. The mean content in the Zhejiang native species was $15.64 \mu \mathrm{g} / \mathrm{g}$, the mean content in the Danxia landform area species was $14.93 \mu \mathrm{g} / \mathrm{g}$, and the mean content in the Guangnan area species was $5.50 \mu \mathrm{g} / \mathrm{g}$. Meanwhile, the concentration of apigenin-6-C- $\beta$-D-xyloside-8-C- $\beta$-Dglucoside in the Zhejiang native species was $31.50 \mu \mathrm{g} / \mathrm{g}$, the concentration in the Danxia landform area species was $46.79 \mu \mathrm{g} / \mathrm{g}$, and the concentration in the Guangnan area species was $18.98 \mu \mathrm{g} / \mathrm{g}$.

\section{HCA analysis}

To investigate the similarities in D. officinale from different sources, we collected 25 batches of samples with 5 representative components, and a cluster analysis was performed. The results are shown in Fig. 5a. The cluster result is consistent with the contents of the components in the different samples. These samples can be divided into 3 categories. D. officinale samples from Zhejiang province, which did not contain rutin or isoviolanthin, were ground together. Samples from Guangxi and

Table 4 Contents [mean \pm SD $(n=3)$ ] of 5 flavonoids in samples 1-25 $(\mu \mathrm{g} / \mathrm{g})$

\begin{tabular}{|c|c|c|c|c|c|}
\hline Samples & Apigenin-6,8-di-C- $\beta$-D-glucoside & Apigenin-6-C- $\beta$-D-xyloside-8-C- $\beta$-D-glucoside & Schaftoside & Isoviolanthin & Rutin \\
\hline GD1 & $68.42 \pm 0.43$ & $41.04 \pm 0.71$ & $9.04 \pm 0.15$ & ND & $606.82 \pm 0.24$ \\
\hline GD2 & $70.07 \pm 0.49$ & $36.33 \pm 0.29$ & $11.37 \pm 0.13$ & ND & $447.91 \pm 1.42$ \\
\hline GD3 & $112.62 \pm 0.24$ & $19.82 \pm 0.37$ & $23.47 \pm 0.26$ & ND & $530.42 \pm 0.42$ \\
\hline$J \times 1$ & $20.62 \pm 0.23$ & $10.01 \pm 0.15$ & $2.31 \pm 0.03$ & ND & $364.25 \pm 2.33$ \\
\hline$J \times 2$ & $42.51 \pm 0.12$ & $34.66 \pm 0.04$ & $18.41 \pm 0.08$ & ND & $155.22 \pm 0.11$ \\
\hline $\mathrm{JX3}$ & $48.36 \pm 0.22$ & $29.75 \pm 0.18$ & $30.82 \pm 0.12$ & ND & $243.81 \pm 0.39$ \\
\hline FJ & $67.64 \pm 0.15$ & $46.74 \pm 0.33$ & $9.14 \pm 0.14$ & ND & $282.28 \pm 0.53$ \\
\hline ZJ1 & $86.63 \pm 0.47$ & $18.48 \pm 0.32$ & $16.61 \pm 0.32$ & ND & ND \\
\hline $\mathrm{ZJ} 2$ & $58.82 \pm 0.43$ & $24.31 \pm 0.47$ & $17.77 \pm 0.32$ & ND & ND \\
\hline ZJ3 & $105.55 \pm 0.68$ & $54.84 \pm 0.15$ & $16.73 \pm 0.31$ & ND & ND \\
\hline ZJ4 & $68.86 \pm 1.28$ & $42.24 \pm 0.84$ & $13.60 \pm 0.39$ & ND & ND \\
\hline ZJ5 & $38.84 \pm 0.81$ & $22.46 \pm 0.45$ & $10.43 \pm 0.09$ & ND & ND \\
\hline ZJ6 & $61.91 \pm 0.36$ & $39.11 \pm 0.55$ & $12.65 \pm 0.04$ & ND & ND \\
\hline ZJ7 & $85.22 \pm 0.81$ & $27.38 \pm 0.80$ & $21.21 \pm 0.54$ & ND & ND \\
\hline ZJ8 & $62.74 \pm 0.20$ & $23.51 \pm 0.16$ & $16.30 \pm 0.47$ & ND & ND \\
\hline YN1 & $75.34 \pm 0.44$ & $21.60 \pm 0.11$ & $4.60 \pm 0.13$ & $164.74 \pm 1.60$ & $10.61 \pm 0.60$ \\
\hline YN2 & $84.70 \pm 0.37$ & $56.32 \pm 0.11$ & $11.43 \pm 0.19$ & $152.96 \pm 0.29$ & $24.81 \pm 0.15$ \\
\hline YN3 & $111.30 \pm 0.31$ & $52.54 \pm 0.27$ & $1.20 \pm 0.11$ & $232.73 \pm 0.34$ & $79.82 \pm 0.21$ \\
\hline YN4 & $36.34 \pm 0.29$ & $12.52 \pm 0.27$ & $5.62 \pm 0.11$ & $42.61 \pm 0.31$ & $13.31 \pm 0.21$ \\
\hline YN5 & $34.73 \pm 0.41$ & $12.08 \pm 0.31$ & $2.91 \pm 0.32$ & $78.83 \pm 0.47$ & $13.87 \pm 0.14$ \\
\hline GX1 & $90.51 \pm 0.37$ & $37.21 \pm 0.12$ & $12.20 \pm 0.09$ & $94.89 \pm 0.74$ & $8.89 \pm 0.81$ \\
\hline $\mathrm{GX} 2$ & $37.10 \pm 0.31$ & $13.53 \pm 0.23$ & $5.30 \pm 0.09$ & $76.77 \pm 0.48$ & $8.31 \pm 0.17$ \\
\hline $\mathrm{GX} 3$ & $37.04 \pm 0.29$ & $13.90 \pm 0.06$ & $5.31 \pm 0.15$ & $76.98 \pm 0.82$ & $8.34 \pm 0.26$ \\
\hline GX4 & $43.51 \pm 0.17$ & $8.52 \pm 0.27$ & $3.32 \pm 0.16$ & $100.25 \pm 0.16$ & $20.12 \pm 0.15$ \\
\hline GX5 & $47.98 \pm 0.28$ & $9.96 \pm 0.24$ & $2.91 \pm 0.12$ & $110.87 \pm 0.60$ & $10.94 \pm 0.27$ \\
\hline
\end{tabular}

Guangdong provinces (No. GD1-GD3), Jiangxi provinces (No. JX1-JX3), Fujian provinces (No. FJ), Zhejiang (No. ZJ1-ZJ8), Yunnan provinces (No. YN1-YN5), Guangxi provinces (No. GX1-GX5)

ND not detected 

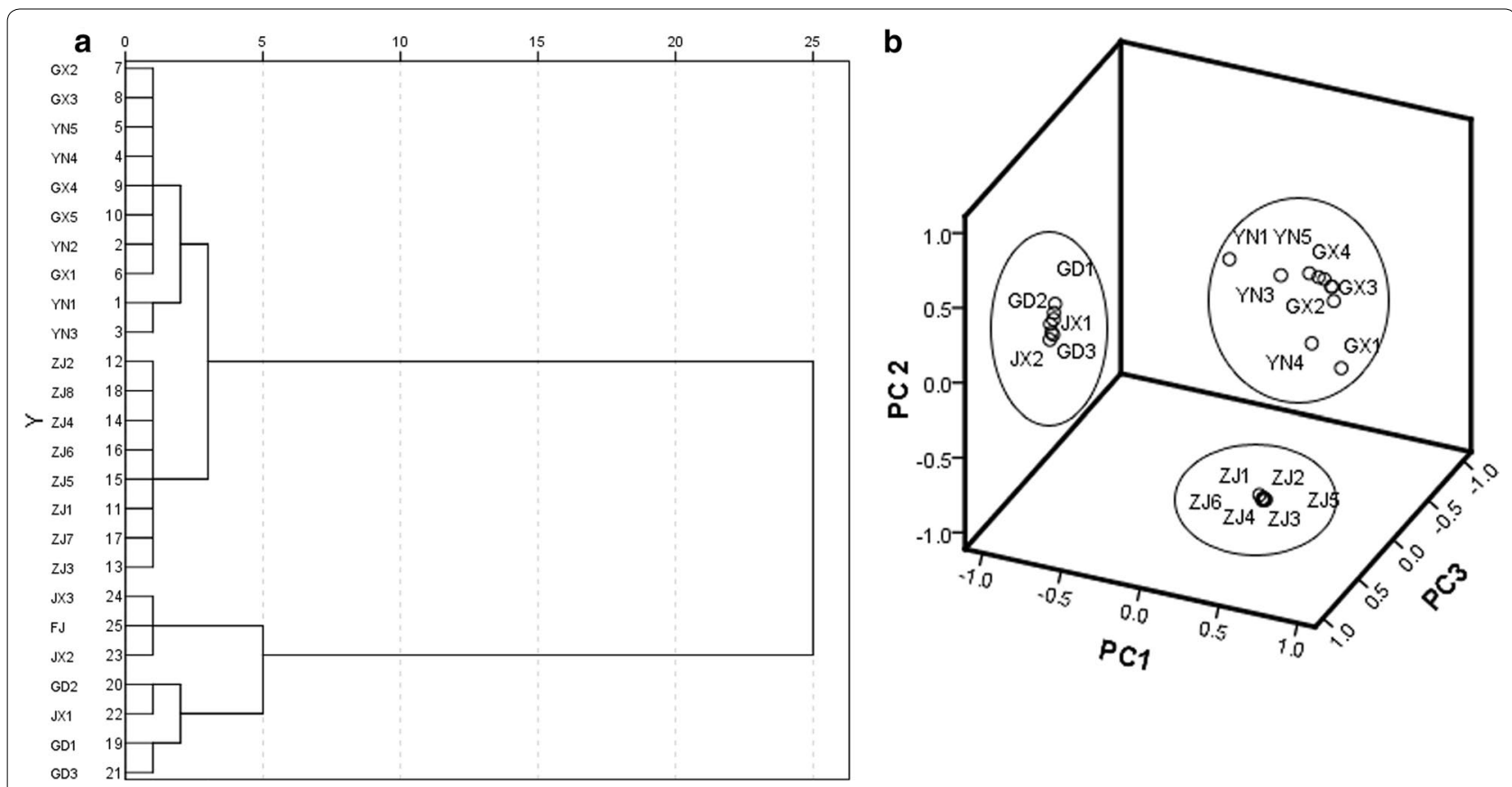

Fig. 5 Dendrogram of hierarchical cluster analysis (HCA) for 25 samples of Dendrobium officinale (a), Principal component analysis (PCA) for 25 samples of Dendrobium officinale (b)

Yunnan provinces were ground together because they both contained isoviolanthin, and samples from Fujian, Guangdong and Jiangxi provinces had a high content of rutin and were ground together. The cluster results agreed with prior studies regarding the major producing regions of $D$. officinale. The presence of isoviolanthin in the samples only from the Yunnan and Guangxi provinces was also confirmed by the results of another research team, which suggests that the sources of our samples were reliable.

\section{PCA analysis}

After importing all data into the SPSS 23.0 software to perform multivariate statistical analysis, the variance contribution rates of the difference components were provided. The variance contribution rate of component 1 was $64.671 \%$, whereas for component 2, it was $19.990 \%$. The variance contribution rate of component 3 was $12.932 \%$. The cumulative variance contribution rate of these three components was $97.593 \%$. The principal component spatial distribution map of the 25 batches of $D$. officinale is shown in Fig. 5b. The samples were ground into three different categories in three-dimensional space. The results were similar to those of the HCA analysis. It is feasible to apply these two methods to process the experimental data to objectively determine the differences in this medicinal material from different producing regions. These methods could be potentially developed to identify $D$. officinale from different producing regions.

\section{Conclusion}

Good standard and good quality are two critical factors for TCM internationalization. The quality of material medicine resources has had a considerable impact on the development of the health industry, which has created a bottleneck for TCMs and has drawn widespread attention. Thus far, the Chinese Pharmacopoeia still uses the contents of mannose as a quality control index of $D$. officinale, which is lacking in specificity. D. officinale is planted in many places in China; however, the Chinese Pharmacopoeia clearly only considers $D$. officinale from one production location as a reference medicine.

In this study, we investigated the chromatographic fingerprint and quantitative analysis of component markers for quality control of $D$. officinale. HCA was used to analyze the samples from different areas, and all samples from the different regions could be grouped into 3 classes. In contrast to another research groups who have used the leaf, which is not the medicinal part of the plant, as the object of their studies or samples from only one location, we collected a large number of $D$. officinale samples from the main producing region in China. The results suggest that there are certain specific flavonoids in samples from different regions of production. We discovered that the contents of apigenin-6,8-di-C- $\beta$-D-glucoside, etc. (five 
representative substances in samples from different locations) exhibited significant differences.

We advise that $D$. officinale from traditional producing locations, such as Zhejiang provinces or the Danxia landform area, should be added as standard medicinal references. Additionally, the chromatographic fingerprint combined with quantification could be applied to distinguish and provide quality control for $D$. officinale samples from different regions of production, which can provide certain references for the Chinese Pharmacopoeia Commission's revised Chinese Pharmacopoeia of quality standards for $D$. officinale.

\section{Abbreviations}

D. officinale: Dendrobium officinale; TCM: Traditional Chinese Medicine; HPLCESI-MS/MS: high performance liquid chromatography-electrospray ionization/ mass spectrometry; $\mathrm{HCA}$ : hierarchical cluster analysis; $t_{\mathrm{R}}$ : retention times.

\section{Author's contributions}

GW proposed research purposes and collected the samples, sum up the conclusion of the study. CZ carried out the identification of flavonoids and drafted the manuscript. ZX and $\mathrm{YH}$ participated in the determination of 5 respectively components. ZL carried out statistical analysis. All authors read and approved the final manuscript.

\section{Competing interests}

The authors declare that they have no competing interests.

\section{Ethics approval and consent to participate}

This article does not contain any studies with human participants or animals performed by any of the author.

\section{Funding}

This study was funded by the Special Foundation of 2017 High Level University Construction (No. A1-AFD018171Z11024) and key project at central government level-the ability establishment of sustainable use for valuable Chinese medicine resources (No. 2060302).

\section{Informed consent}

Informed consent was obtained from all individual participants included in the study.

\section{Publisher's Note}

Springer Nature remains neutral with regard to jurisdictional claims in published maps and institutional affiliations.

Received: 1 November 2017 Accepted: 10 March 2018

Published online: 12 April 2018

\section{References}

1. Zhang J, He C, Wu K et al (2016) Transcriptome analysis of dendrobium officinale and its application to the identification of genes associated with polysaccharide synthesis. Front Plant Sci 7:5

2. Zhang G, Xu Q, Bian C et al (2016) The Dendrobium catenatum Lindl. genome sequence provides insights into polysaccharide synthase, floral development and adaptive evolution. Sci Rep 6:19029
3. Tong L, Wang L, Zhou X et al (2016) Antitumor activity of Dendrobium devonianum polysaccharides based on their immunomodulatory effects in S180 tumor-bearing mice. RSC Adv 6:40250-40257

4. Zheng S, Wu H, Li Z et al (2015) Ultrasound/microwave-assisted solidliquid-solid dispersive extraction with high-performance liquid chromatography coupled to tandem mass spectrometry for the determination of neonicotinoid insecticides in Dendrobium officinale. J Sep Sci 38:121-127

5. Xu J, Han Q, Li S et al (2013) Chemistry, bioactivity and quality control of Dendrobium, a commonly used tonic herb in traditional Chinese medicine. Phytochem Rev 12:341-367

6. Huang X, Nie S, Cai H et al (2015) Study on Dendrobium officinale O-acetyl-glucomannan (Dendronan): part IV. immunomodulatory activity in vivo. J Funct Foods 15:525-532

7. Huang X, Nie S, Cai H et al (2015) Study on Dendrobium officinale O-acetyl-glucomannan (Dendronan (R)): part VI. protective effects against oxidative stress in immunosuppressed mice. Food Res Int 72:168-173

8. Pan L, Li X, Wang M et al (2014) Comparison of hypoglycemic and antioxidative effects of polysaccharides from four different Dendrobium species. Int J Biol Macromol 64:420-427

9. Wu Y, Dong Z, Wu H et al (2014) Comparative studies on Ophiopogonis and Liriopes based on the determination of 11 bioactive components using LC-MS/MS and hierarchical clustering analysis. Food Res Int $57: 15-25$

10. Luo L, Zhu M, Chen L, Yang B, Shao X (2013) Chemical constituents of Dendrobium officinale Kimura et Migo. Chin Pharm J 48:1681-1683

11. Huang K, Li Y, Tao S et al (2016) Purification, characterization and biological activity of polysaccharides from Dendrobium officinale. Molecules. https://doi.org/10.3390/molecules21060701

12. Chen $X$, Wang F, Wang $Y$ et al (2012) Discrimination of the rare medicinal plant Dendrobium officinale based on naringenin, bibenzyl, and polysaccharides. Sci China Life Sci 55:1092-1099

13. Xu J, Li S, Yue R et al (2014) A novel and rapid HPGPC-based strategy for quality control of saccharide-dominant herbal materials: Dendrobium officinale, a case study. Anal Bioanal Chem 406:6409-6417

14. Bin Y, Wang Y, An X, Ren J (2014) Dendrobium polysaccharide purification and determination of monosaccharide composition. Sci Technol Food Ind 35:122-125

15. Xin M, Zhang E, Li N et al (2013) Effects of different drying methods on polysaccharides and dendrobine from Dendrobium candidum. J S Agric 44:1347-1350

16. Song M, Li G, Chen X, Li Y, Li X (2013) Primary study of Dendrobium polysaccharides improving immunity activity on mouse. Chin Pharm J 48:428-430

17. Cao J, Xia X, Chen X, Xiao J, Wang Q (2013) Characterization of flavonoids from Dryopteris erythrosora and evaluation of their antioxidant, anticancer and acetylcholinesterase inhibition activities. Food Chem Toxicol 51:242-250

18. Kroslakova I, Pedrussio S, Wolfram E (2016) Direct coupling of HPTLC with MALDI-TOF MS for qualitative detection of flavonoids on phytochemical fingerprints. Phytochem Anal 27:222-228

19. Liu W, Kong Y, Zu Y et al (2010) Determination and quantification of active phenolic compounds in pigeon pea leaves and its medicinal product using liquid chromatography-tandem mass spectrometry. J Chromatogr A 1217:4723-4731

20. Tao Y, Cai H, Li W, Cai B (2015) Ultrafiltration coupled with high-performance liquid chromatography and quadrupole-time-of-flight mass spectrometry for screening lipase binders from different extracts of Dendrobium officinale. Anal Bioanal Chem 407:6081-6093

21. Ye Z, Dai J, Zhang C et al (2017) Chemical differentiation of Dendrobium officinale and Dendrobium devonianum by using HPLC fingerprints, HPLC-ESI-MS, and HPTLC analyses. Evid Based Complement Alternat Med 2017:8647212

22. Wei G, Shun Q, Huang Y et al (2014) Comparative study of HPLC characteristic spectrum of Dendrobium officinale from three provenance sources. Tradit Chin Drug Res Clin Pharmacol 25:467-471 